\title{
CONTABILIDAD ELECTRÓNICA, ENFOQUE ESENCIAL PARA LA EDUCACIÓN CONTABLE
}

\section{ELECTRONIC ACCOUNTING, ESSENTIAL APPROACH TO ACCOUNTING EDUCATION}

Eduardo Pérez Sánchez*, Aransazú Ávalos Díaz**

*Licenciado en Contaduría Pública. Universidad Juárez Autónoma de Tabasco.

**Doctora en Educación y Maestra en Administración de Empresa. Universidad Juárez Autónoma de Tabasco. Email: aransazu.avadi@gmail.com

Dirección para recibir correspondencia: edukavia@gmail.com 


\section{RESUMEN}

OBJETIVO: Dar a conocer la base e importancia que tiene la contabilidad electrónica como una nueva y obligatoria modalidad de contabilizar, resaltando la interrelación que hay entre educación y tecnología, formándose día con día como parte de una nueva cultura académica.

MATERIAL Y MÉTODO: Esta investigación es de tipo cuantitativa, transversal y retrospectiva. Se seleccionaron 18 grupos de alumnos de la Universidad Juárez Autónoma de Tabasco, obteniendo un total de 522 estudiantes, los cuales constituyeron parte de nuestra población y los resultados obtenidos se plasmaron en gráficas.

RESULTADOS: 308 estudiantes que formaron parte del 59\% de la muestra, asimismo, en la investigación no tienen conocimiento de qué es y cómo funciona la contabilidad electrónica.

CONCLUSIÓN: Dada la obligatoriedad de la contabilidad electrónica, surge la necesidad que el plan de estudio de la Licenciatura en Contaduría, incluya la enseñanza de este tema; ayudando así a los egresados con su desarrollo profesional, con el propósito de obtener mayores oportunidades, tanto en las organizaciones como en cualquier otro campo.

PALABRAS CLAVE: Cultura académica. Organizaciones. Desarrollo profesional. Educación. Tecnología.

\section{ABSTRACT}

OBJECTIVE: To make known the basis and importance of electronic accounting as a new and mandatory way of counting, highlighting the interrelation between education and technology, becoming day by day as part of a new academic culture.

MATERIAL AND METHOD: This research is a quantitative, transversal and retrospective type. 18 groups of students from the Universidad Juárez Autónoma de Tabasco were chosen, obtaining a total of 522 students, who were part of the population and the results obtained were shown in graphs. 
RESULTS: 308 students, who were part of the 59\% of the sample, likewise in the research, have no knowledge about what electronic accounting is and how it works.

CONCLUSION: Given the mandatory nature of electronic accounting, it arises the need that the syllabus of the bachelor's degree in accounting includes the teaching of this topic in order to help graduates with their professional development, with the purpose of obtaining better opportunities, in both, organizations and any other field.

KEY WORDS: Academic culture. Organizations. Professional development. Education. Technology.

\section{INTRODUCCIÓN}

“El comienzo de las prácticas contables se remonta desde tiempos muy antiguos, cuando el hombre se ve obligado a llevar registros y controles de sus propiedades porque su memoria no bastaba para guardar la información requerida" (Chávez, 2015, p. 73).

La práctica contable se ha ido transformando hasta nuestros tiempos, llegando a convertirse en una técnica inclusive nombrado por Lara (2015), una ciencia que enseña las normas y procedimientos para ordenar, analizar y registrar las operaciones practicadas por unidades económicas.

La innovación es un cambio que alcanza a nuestro entorno diariamente, la tecnología es la principal causante de que esto suceda a través de nuevas invenciones, métodos y técnicas.

Claramente, la contabilidad no está exenta y actualmente, el modo de contabilizar y las leyes fiscales se mantienen en constante cambio, adaptándose a las tecnologías habientes.

Lee (2017) considera que, nuestro país se encuentra en ese proceso de transformación de los servicios electrónicos y se puede destacar que la autoridad fiscal, cuenta con un portal a través del cual se hace la mayoría de trámites hacendarios. 
CONTABILIDAD ELECTRÓNICA, ENFOQUE ESENCIAL PARA LA EDUCACIÓN CONTABLE

Respecto a esto, Ceballos (2015) explica que, la autoridad tributaria busca dotarse de una red de información estratégica para así determinar o presumir, delito de defraudación fiscal y oportunidades de recaudación potencial y para llevar a cabo este proyecto tiene como pilar la información electrónica.

El anexo de asignaturas que expliquen y enseñen las nuevas modalidades contables y tecnológicas, es de vital ayuda para el desarrollo de los conocimientos de los alumnos, actualmente el Plan de Estudios que se está implementado en la licenciatura, tiene tan solo una materia, el cual toca un punto importante en la contabilidad electrónica, teórica y práctica, como lo son los software contables, dejando en una brecha lo extenso que es, este nuevo modo de registro y manejo de la información contable.

Es por ello, que para los estudiantes de Licenciatura en Contaduría, es de vital ayuda tener conocimiento necesario del actuar de los mecanismos de manejo de datos financieros en la contabilidad electrónica, sus programas, funciones y su alcance, para estar preparados y que puedan enfrentarse a los problemas en la práctica laboral y sean capaz de satisfacer las necesidades que se vayan desplegando en la sociedad.

\section{MARCO TEÓRICO}

\section{¿Qué es la Contabilidad en México?}

Pérez y Fol (2016) nos dicen que:

Las Normas de Información Financiera (NIF) en su apartado A-1, Estructura de las Normas de Información Financiera, define el concepto de contabilidad en los términos siguientes: La contabilidad es una técnica que se utiliza para el registro de las operaciones que afectan económicamente a una entidad y que produce sistemática y estructuradamente información financiera. Las operaciones que afectan económicamente a una entidad incluyen las transacciones, transformaciones internas y otros eventos.

De donde se desprende que:

1. Es una técnica,

2. Se utiliza para el registro de las operaciones, y

3. Produce sistemática y estructuradamente información financiera (p. 19). 
CONTABILIDAD ELECTRÓNICA, ENFOQUE ESENCIAL PARA LA EDUCACIÓN CONTABLE

Lang (2015) explica que, la contabilidad tiene la responsabilidad de promover el bienestar humano, al brindar información para que las empresas puedan contribuir al desarrollo sostenible.

En términos breves, la contabilidad es una técnica para registrar las operaciones realizadas por una organización, y con esto generar información financiera que sea útil para la toma de decisiones económicas.

\section{Obligación legal}

"La aplicación de la técnica contable es una obligación jurídica que va más allá de las leyes fiscales. De su correcta aplicación resultará, la determinación del justo pago de contribuciones y el cumplimiento de diversos ordenamientos" (Marín, 2014, p. 45).

Las bases legales que tienen las empresas al llevar un registro contable. En México, se hallan en las siguientes leyes:

- $\quad$ En el código de comercio: Capítulo III Articulo 33 al 38.

- En la ley del impuesto sobre la renta: Título II, Capítulo VIII Artículo 58.

- En el código fiscal de la federación: Título II, Artículo 28.

- En el reglamento del código fiscal de la federación: Sección III, Artículo 26 al 29.

- En la ley del impuesto al valor agregado: Capítulo VII, Artículo 32 Fracción I.

- En el reglamento de la ley del impuesto al valor agregado: Capítulo VII, Artículo 46.

El gobierno, con el fin de inspeccionar la correcta práctica del pago de impuestos, acuerda como obligación que toda persona física o moral que ejecute ciertas actividades comerciales lleve una contabilidad.

\section{La contabilidad, de lo manual a lo electrónico}

El registrar la contabilidad es una obligación de carácter fiscal; su origen se remonta al Decreto del 30 de abril de 1959 en el que se establece la revisión, investigación y vigilancia al cumplimiento de las obligaciones fiscales de los contribuyentes. Este introduce la presentación y entrega a la autoridad hacendaria de los registros y asientos contables para efectos fiscales. En este sentido, se autorizaba a los contadores públicos a formular dictámenes sobre los estados financieros de los contribuyentes y llevar el registro y control (Secretaría de Hacienda y Crédito Público [SHCP], 2015, p. 36). 
CONTABILIDAD ELECTRÓNICA, ENFOQUE ESENCIAL PARA LA EDUCACIÓN CONTABLE

Los contadores empleaban libros de contabilidad separados para cada área del sistema de contabilidad, como cuentas por cobrar, cuentas por pagar y ventas; posteriormente consolidaban estos libros en un libro mayor, obteniendo el saldo de cada libro. Para crear estados financieros usaban el cuaderno del libro general y con ello, proceder con los cálculos de impuestos y obligaciones.

La demanda para disponer de estrategias de soluciones tecnológicas a los negocios para la administración y control de sus operaciones, propició a su vez el surgimiento de software y programas para el registro de la contabilidad y la administración de los recursos financieros, tanto para empresas grandes como para pequeños emprendedores, situación que favoreció la regulación en las disposiciones fiscales sobre el uso de la contabilidad electrónica al definir los parámetros y criterios tecnológicos para realizarla (SHCP, 2015, p. 36). Fue así, como a partir de 2014 de forma legal en el CFF, se estableció la obligación de registrar la información contable en medios electrónicos.

\section{¿Qué es la contabilidad electrónica?}

Respecto a este tema, Galindo y Hernández (2017), dice lo siguiente:

Esta denominación se ha popularizado y parece ser un distante concepto de aquel que los contadores hacen día con día; ha generado confusión entre los profesionales de la contaduría por todas las voces que se han levantado; sin embargo, no es más que la introducción de la tecnología en el diario que hacer, lo cual se resume en dos expresiones:

A. Automatización de los procesos; es la utilización de sistemas tecnológicos el cual busca hacer más fácil, efectivo y eficiente la actividad de contabilizar.

B. XML; es un archivo que contiene el formato y la sintaxis fiscalmente válida para la representación digital de facturas electrónicas.

La modificación busca otorgar mayores elementos al brazo fiscalizador del gobierno para que en caso de solicitar información, el contribuyente deba entregar todos los documentos que se listan en el CFF y su Reglamento (p. 57).

La contabilidad electrónica es el deber de llevar los movimientos y asientos contables por medios electrónicos, siguiendo con la entrega periódica de la información contable a través del portal de internet del Sistema de Administración Tributaria (SAT). 
CONTABILIDAD ELECTRÓNICA, ENFOQUE ESENCIAL PARA LA EDUCACIÓN CONTABLE

Corvera (2014) dice que, por medio de este mecanismo, la autoridad fiscal podrá notificar, requerir y recibir documentación del contribuyente.

Con esto, se planean en forma más organizada las revisiones del fisco y se garantiza una sola contabilidad de los contribuyentes.

\section{Inducción legal de la contabilidad electrónica}

"Mediante la reforma que sufrió el artículo 28 del CFF, a partir del 1ro de enero de 2014, los registros o asientos que integran la contabilidad sólo se llevarán en medios electrónicos conforme lo establezcan el CFF y las disposiciones de carácter general" (Pérez y Fol, 2016, p. 39).

Ceballos (2015), comenta lo siguiente:

Todas las personas morales y las personas físicas con actividades empresariales y profesionales, así como algunos arrendadores, están obligados a llevar contabilidad de acuerdo a la Ley del ISR, haciendo los registros de acuerdo a lo establecido en el CFF.

Artículo 28. Las personas que de acuerdo con las disposiciones fiscales estén obligadas a llevar contabilidad, estarán a lo siguiente:

I. La contabilidad, para efectos fiscales, se integra por los libros, sistemas y registros contables, papeles de trabajo, estados de cuenta, cuentas especiales, libros y registros sociales, control de inventarios y método de valuación, discos y cintas o cualquier otro medio procesable de almacenamiento de datos, los equipos o sistemas electrónicos de registro fiscal y sus respectivos registros, además de la documentación comprobatoria de los asientos respectivos, así como toda la documentación e información relacionada con el cumplimiento de las disposiciones fiscales, la que acredite sus ingresos y deducciones, y la que obliguen otras leyes.

II. Los equipos y programas informáticos para llevar los controles volumétricos serán aquellos que autorice para tal efecto el Servicio de Administración Tributaria, los cuales deberán mantenerse en operación en todo momento.

III. Los registros o asientos contables a que se refiere la fracción anterior deberán cumplir con los requisitos que establezca el Reglamento de este Código y las disposiciones de carácter general que emita el Servicio de Administración Tributaria. 
IV. Los registros o asientos que integran la contabilidad se llevarán en medios electrónicos conforme lo establezcan el Reglamento de este Código y las disposiciones de carácter general que emita el Servicio de Administración Tributaria. La documentación comprobatoria de dichos registros o asientos deberá estar disponible en el domicilio fiscal del contribuyente.

V. Ingresarán de forma mensual su información contable a través de la página de Internet del SAT, de conformidad con reglas de carácter general que se emitan para tal efecto" (p.25).

Las personas que estén obligadas a llevar contabilidad, tendrán que cumplir con los requisitos que establezca el Reglamento del CFF y las disposiciones de carácter general que emita el SAT respecto a los registros o asientos contables que hagan; también menciona que los registros 0 asientos que integran la contabilidad se llevarán en medios electrónicos, además que deberán estar disponibles en el domicilio fiscal del contribuyente, y por último, que Ingresarán de forma mensual su información contable a través de la página de Internet del SAT.

“Mediante la Resolución Miscelánea Fiscal para 2014, el 1 de julio del mismo año se establece el cumplimiento de la obligación a que se refiere el artículo 28 del Código, es decir, llevar los registros o asientos que integran la contabilidad en medios electrónicos (contabilidad electrónica) conforme lo establezcan el CFF y las reglas misceláneas que emita el SAT" (Pérez y Fol, 2016, p. 41).

Según Galindo y Hernández (2017), La Miscelánea obliga a lo siguiente:

I. Los papeles de trabajo relativos al cálculo de la deducción de inversiones, relacionándola con la documentación comprobatoria que permita identificar la fecha de adquisición del bien, su descripción, el monto original de la inversión, el porcentaje e importe de su deducción anual, son parte de la contabilidad.

II. El registro de los asientos contables establecido en la fracción I Apartado B, se podrá efectuar dentro del mes siguiente a la fecha en que se realicen las actividades respectivas.

III. En caso de no contar con la información que permita identificar el medio de pago, se podrá incorporar en los registros, la expresión "NA", sin especificar si fue de contado, a crédito o en parcialidades, y el medio de pago o de extinción de dicha obligación, según corresponda (p. 87). 
CONTABILIDAD ELECTRÓNICA, ENFOQUE ESENCIAL PARA LA EDUCACIÓN CONTABLE

"Los contribuyentes que lleven su contabilidad utilizando registros electrónicos deberán proporcionárselas a las autoridades fiscales cuando así lo soliciten en los medios procesables que utilicen, la información sobre sus clientes y proveedores, así como aquella relacionada con su contabilidad que tengas dichos medios" (Lara, 2006, p. 245).

\section{Ventajas de la contabilidad electrónica}

Adoptar servicios electrónicos era una prioridad, cuyos beneficios no podrían apreciarse sin un cambio en cuanto a su forma y organización. Entonces, se asumió un esquema que agilizara la gestión interna, este esquema de diversificación de los servicios tributarios representa ventajas tanto para el contribuyente como para la administración tributaria entre las que se encuentran las siguientes:

- Múltiples opciones de entrega para el contribuyente.

- Elimina recursos destinados al empleo del papel y hace más eficiente el manejo de archivos digitales electrónicos (SHCP, 2015, p. 10).

- Reducción de tiempo y costos, una vez registradas las operaciones, la elaboración de los auxiliares, estados financieros, entre otros, la computadora los realiza en un proceso automático en tan solo fracciones de segundo, lo cual representa un gran ahorro de tiempo (Lara, 2006, p. 245).

Las herramientas electrónicas tienen la función de tomar los comprobantes y cargarlos en el sistema contable para que sea más práctico el asiento contable.

"Sin embargo, aún se siguen utilizando documentos en papel, por lo que se recomienda digitalizarlos, para establecer un sistema adecuado de almacenamiento en medios electrónicos para cuando la autoridad pretenda conocer el contenido de este tipo de soportes contables como actas, cheques" (Castro, 2015, p. 21).

La contabilidad electrónica es una normatividad obligatoria y la evolución de todo lo que antes un contador público hacía de modo manual; pólizas de diario, egresos, estados financieros, declaraciones.

Es por ello, que para los estudiantes de la Licenciatura en contaduría es de vital importancia tener conocimiento de dicha evolución. 
CONTABILIDAD ELECTRÓNICA, ENFOQUE ESENCIAL PARA LA EDUCACIÓN CONTABLE

Tabla 1

Asignaturas del área general, del Plan de Estudios de la Licenciatura en Contaduría Pública

\begin{tabular}{|c|c|c|c|c|c|}
\hline Clave & $\begin{array}{l}\text { Nombre de la } \\
\text { asignatura }\end{array}$ & HT & $\mathrm{HP}$ & C & Tipo \\
\hline F1009 & $\begin{array}{l}\text { Herramientas de } \\
\text { Computo }\end{array}$ & & 4 & 4 & Obl \\
\hline F1005 & Lengua Extranjera & 1 & 2 & 4 & Obl \\
\hline BF1018 & $\begin{array}{l}\text { Informática para la } \\
\text { Gestión y } \\
\text { Manipulación de } \\
\text { Datos }\end{array}$ & 1 & 3 & 5 & Obl \\
\hline BF1019 & $\begin{array}{l}\text { Introducción a la } \\
\text { Estadística }\end{array}$ & 1 & 3 & 5 & Obl \\
\hline BF1028 & Microeconomía & 1 & 3 & 4 & Obl \\
\hline F1006 & Lectura y Redacción & 1 & 3 & 5 & Obl \\
\hline BF1010 & Contabilidad & 1 & 4 & 6 & Obl \\
\hline F1008 & $\begin{array}{l}\text { Pensamiento } \\
\text { Matemático }\end{array}$ & 1 & 4 & 6 & Obl \\
\hline F1001 & Ética & 2 & 1 & 5 & Obl \\
\hline F1002 & Filosofía & 2 & 1 & 5 & Obl \\
\hline F1003 & Metodología & 2 & 1 & 5 & Obl \\
\hline F1004 & Cultura Ambiental & 2 & 1 & 5 & Obl \\
\hline F1007 & Derechos Humanos & 2 & 1 & 5 & Obl \\
\hline BF1017 & Mercadotecnia & 2 & 2 & 6 & Obl \\
\hline BF1030 & $\begin{array}{l}\text { Administración y } \\
\text { Gestión Estratégica }\end{array}$ & 2 & 2 & 6 & Obl \\
\hline BF1027 & $\begin{array}{l}\text { Introducción al } \\
\text { Derecho }\end{array}$ & 3 & 1 & 7 & Obl \\
\hline
\end{tabular}

Fuente: Plataforma web de la UJAT. 
Tabla 2

Asignaturas del área sustantiva profesional

\begin{tabular}{|c|c|c|c|c|c|}
\hline Clave & Nombre de la asignatura & $\mathrm{HT}$ & HP & $\mathrm{C}$ & Tipo \\
\hline BF1011 & Análisis e Interpretación de Estados Financieros & 1 & 3 & 5 & Obl \\
\hline BF1012 & Matemáticas Financieras & 1 & 3 & 5 & Obl \\
\hline BF1013 & Administración Financiera & 1 & 3 & 5 & Obl \\
\hline BF1016 & Desarrollo de Emprendedores & 1 & 3 & 5 & Obl \\
\hline BF1020 & Estadística Inferencial & 1 & 3 & 5 & Obl \\
\hline BF1023 & Inglés de Negocios I & 1 & 3 & 5 & Obl \\
\hline BF1024 & Inglés de Negocios II & 1 & 3 & 5 & Obl \\
\hline BF1025 & Ingles de Negocios III & 1 & 3 & 5 & Obl \\
\hline BF1037 & Investigación de Operaciones & 1 & 3 & 5 & Obl \\
\hline BF1038 & Inglés de Negocios IV & 1 & 3 & 5 & Obl \\
\hline BF1044 & Derecho Laboral & 1 & 3 & 5 & Obl \\
\hline BF1035 & Derecho Mercantil & 1 & 4 & 6 & Obl \\
\hline BF1100 & Métodos de Valuación de Inventarios y Sistema & 1 & 4 & 6 & Obl \\
\hline BF1101 & Activo y Pasivo & 1 & 4 & 6 & Obl \\
\hline BF1102 & Patrimonio y Capital Contable & 1 & 4 & 6 & Obl \\
\hline BF1103 & Elaboración y Evaluación de Estados Financieros & 1 & 4 & 6 & Obl \\
\hline BF1107 & Costos Históricos - Reales & 1 & 4 & 6 & Obl \\
\hline BF1108 & Elaboración de Presupuestos & 1 & 4 & 6 & Obl \\
\hline BF1109 & Costos Predeterminados & 1 & 4 & 6 & Obl \\
\hline BF1113 & Auditoría Fiscal & 1 & 4 & 6 & Obl \\
\hline BF1031 & Comportamiento Organizacional & 2 & 2 & 6 & Obl \\
\hline BF1032 & Administración del Factor Humano I & 2 & 2 & 6 & Obl \\
\hline BF1123 & Informática Aplicada a la Contabilidad & 2 & 2 & 6 & Obl \\
\hline BF1104 & $\begin{array}{l}\text { Consolidación de Estados Financieros, Fusión y } \\
\text { Escisión }\end{array}$ & 2 & 3 & 7 & Obl \\
\hline BF1110 & Técnicas de Costos & 2 & 3 & 7 & Obl \\
\hline BF1112 & Auditoría Financiera & 2 & 3 & 7 & Obl \\
\hline BF1118 & Impuestos Indirectos & 2 & 3 & 7 & Obl \\
\hline BF1119 & Impuestos Directos Personas Morales & 2 & 3 & 7 & Obl \\
\hline BF1120 & Impuestos Directos Personas Físicas & 2 & 3 & 7 & Obl \\
\hline BF1121 & Seguridad Social e Impuestos Especiales & 2 & 4 & 8 & Obl \\
\hline BF1111 & Principios Básicos de Auditoría & 3 & 2 & 8 & Obl \\
\hline BF1117 & Fundamentos del Derecho Fiscal & 3 & 2 & 8 & Obl \\
\hline
\end{tabular}

Fuente: Plataforma web de la UJAT. 
CONTABILIDAD ELECTRÓNICA, ENFOQUE ESENCIAL PARA LA EDUCACIÓN CONTABLE

Tabla 3

Asignaturas del área integral profesional

\begin{tabular}{clcccc}
\hline Clave & \multicolumn{1}{c}{ Nombre de la asignatura } & HT & HP & C & Tipo \\
\hline BF1045 & Auditoría Gubernamental & 1 & 3 & 5 & Obl \\
BF1106 & Contabilidades Especiales & 1 & 3 & 5 & Obl \\
& Formulación y Evaluación de Proyectos de & 1 & 3 & 5 & Obl \\
BF1124 & Inversión & 1 & 4 & 6 & Obl \\
BF1127 & Presupuestos Gubernamentales & 2 & 2 & 6 & Obl \\
BF1105 & Contabilidad Internacional & 2 & 3 & 7 & Obl \\
BF1114 & Auditoria Interna y Operacional & 1 & 3 & 5 & Obl \\
& Optativa 1 & 1 & 3 & 5 & Obl \\
& Optativa 2 & 1 & 3 & 5 & Obl \\
& Optativa 3 & 1 & 3 & 5 & Obl \\
& Optativa 4 & & & & \\
\hline
\end{tabular}

Fuente: Plataforma web de la UJAT.

Tabla 4

Asignaturas del área de formación transversal

\begin{tabular}{|c|c|c|c|c|c|}
\hline Clave & Nombre de la asignatura & HT & $\mathrm{HP}$ & $\mathrm{C}$ & Tipo \\
\hline BF1021 & Protocolo de Investigación & & 3 & 3 & Obl \\
\hline BF1022 & Seminario de Titulación & & 3 & 3 & Obl \\
\hline F1999 & Servicio Social & & 10 & 10 & Obl Ext \\
\hline BF1998 & Prácticas Profesionales & & & 8 & Obl Ext \\
\hline
\end{tabular}

Fuente: Plataforma web de la UJAT.

En la tabla 1, (Asignaturas del área general) puede apreciarse la materia llamada "Informática para la Gestión y Manipulación de Datos", la cual aborda en general el uso de programas informáticos como Excel para el manejo de datos, algo que en proceso de la contabilidad actual es básico. En la tabla 2 (Asignaturas del área sustantiva profesional) se encuentra la materia que toca apenas un poco acerca de la contabilidad electrónica, "Informática Aplicada a la Contabilidad", la cual enseña el modo de uso de los software contables como Contpaq y Aspel.

De igual manera, dos asignaturas del Plan de Estudios hablan ligeramente de la contabilidad electrónica, algo critico respecto a la modalidad actual acerca de los procesos contables. 


\section{MATERIAL Y MÉTODO}

Con base en la necesidad de preparar a los alumnos para satisfacer las necesidades de la sociedad actual, nace la incógnita de saber si los estudiantes que están a mitad de la carrera universitaria, tienen el conocimiento necesario acerca de la contabilidad electrónica. Para obtener una respuesta, se encuestó a 522 alumnos a partir del quinto semestre de la Licenciatura en Contaduría de la División Académica de Ciencias Económico Administrativas en la Universidad Juárez Autónoma de Tabasco, obteniendo de esta una investigación de tipo cuantitativa, no experimental, descriptiva, transversal y prospectiva.

\section{RESULTADOS}

A continuación, se presentan y detallan los resultados más destacados de dicha investigación:

Del $100 \%$ de alumnos encuestados en la Universidad, el $57 \%$ no sabe qué es la contabilidad electrónica, el $28 \%$ dijo saber muy poco y tan solo el 15\% conoce esta modalidad (ver figura 1).

\section{¿Sabes con seguridad qué es la contabilidad electrónica?}

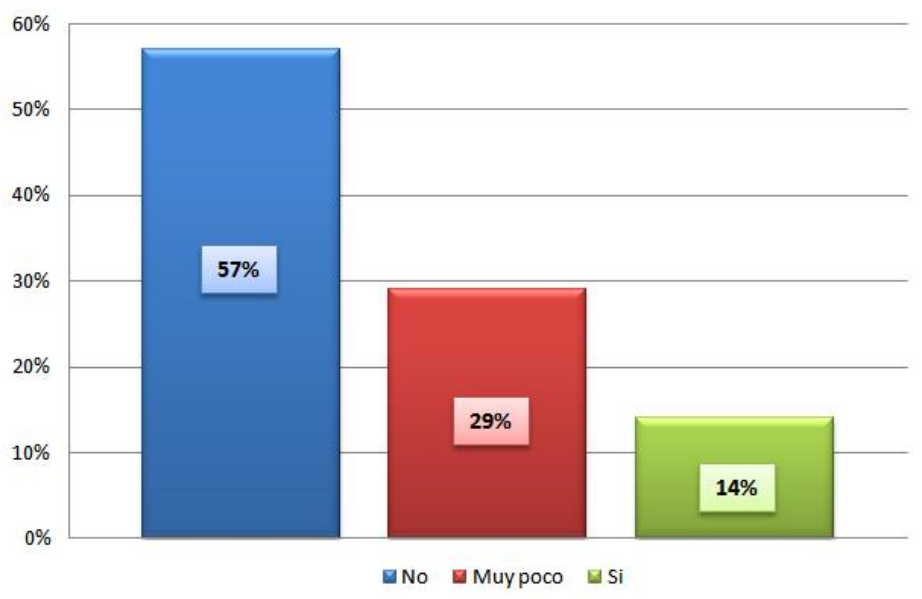

Figura 1. Se refleja que más del $50 \%$ de alumnos, no sabe lo básico acerca de esta modalidad, como lo es su concepto.

Fuente: Resultado de la investigación.

Saber que es y de que se trata un tema en específico es lo primordial para poder aprenderlo, el concepto y la teoría según Granada (1984) es el proceso de generación y construcción de conocimientos. 
Con respecto, si poseen conocimiento del año en que se incorporó como obligación la contabilidad electrónica en las empresas, el $86 \%$ dijo no saber y sólo el 14\% dijo tener conocimiento de ello (ver figura 2).

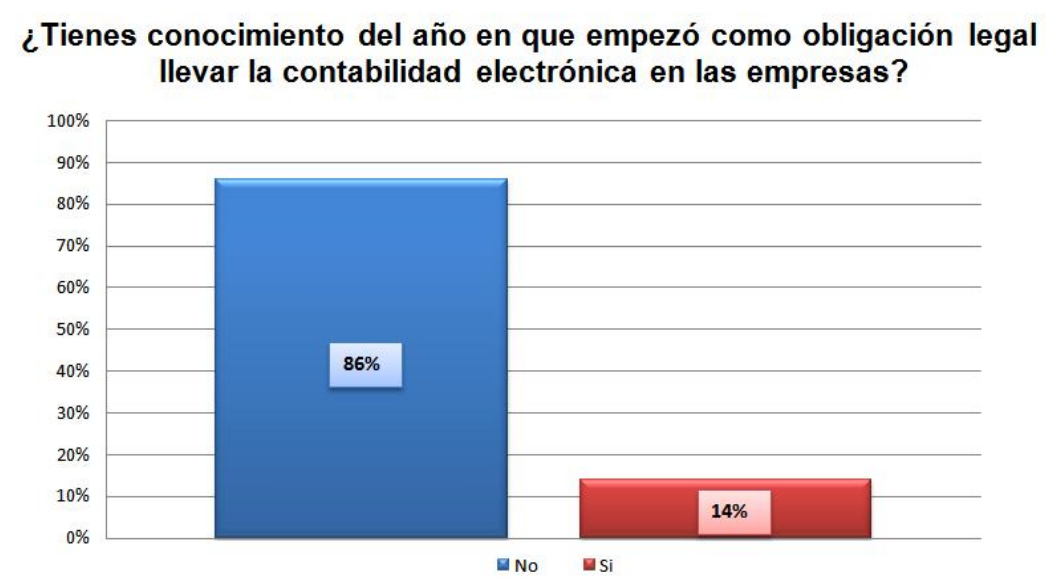

Figura 2. La mayor parte de alumnos no saben la fecha del nacimiento legal de este mecanismo, que es obligatorio actualmente.

Fuente: Resultado de la investigación.

A partir del mes de enero del año 2014, en el CFF queda como obligación para todas las personas físicas y morales llevar sus registros contables en medios electrónicos, para su posterior entrega e inspección. Los estudiantes deben tener en cuenta este tipo de fundamentos, ya que en la mayoría de empresas se manejan datos históricos y llegan a existir casos donde se necesita información pasada para llevar a cabo alguna actividad.

Conforme al rubro fiscal, se les cuestionó si saben manejar la página web del Servicio de Administración Tributaria (SAT) para algún tipo de declaración, el 68\% dijo no saber, el 22\% señaló saber muy poco y el 10\% afirmó saber utilizar la página web para asuntos fiscales (ver figura 3). 
¿Sabes cómo utilizar la página del SAT para algún tipo de declaración fiscal?

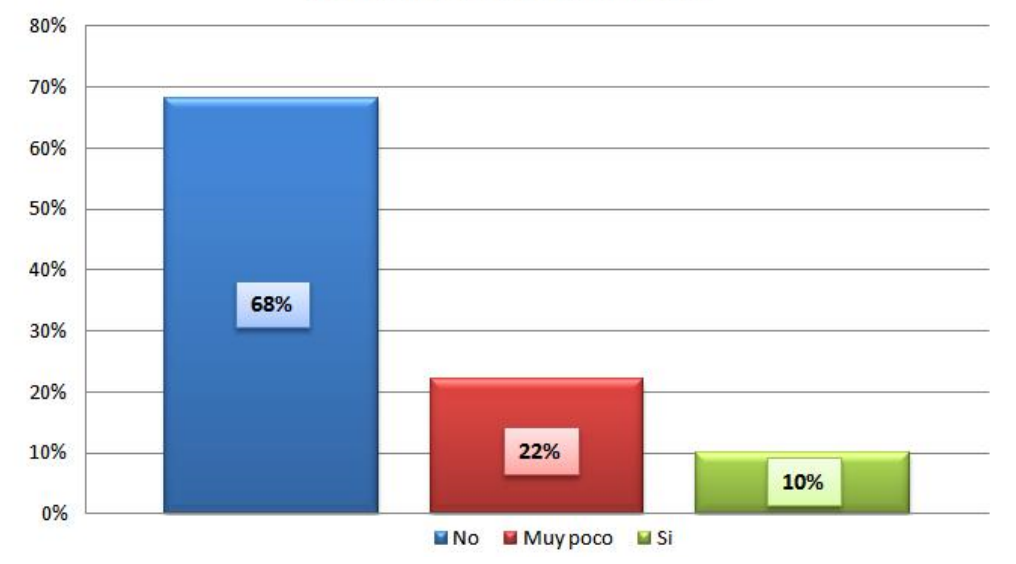

Figura 3. Más de la mitad de los alumnos no tienen conocimiento de cómo funciona la página web del fisco para el cumplimiento de obligaciones hacendarias.

Fuente: Resultado de la investigación.

Periódicamente, el contribuyente debe enviar su información contable a través del portal del SAT vía internet, este es un proceso que necesita de contadores públicos preparados, con la debida noción del mecanismo de esta misma.

Respecto a lo contable, se preguntó si saben utilizar los softwares especializados para contabilizar, el $45 \%$ dijo sí, el 30\% dijo saber muy poco, y el $25 \%$ dijo no saber utilizar algún software (ver figura 4).

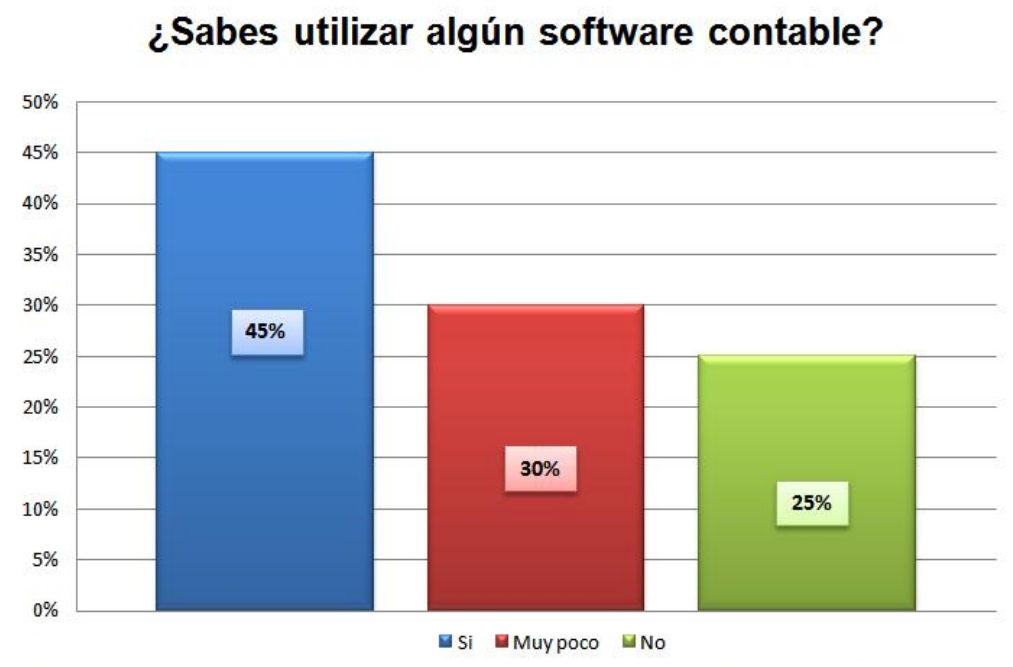

Figura 4. La mayor parte de los alumnos saben utilizar al menos un software contable, debido a algunas de las asignaturas del Plan de Estudios de la universidad.

Fuente: Resultado de la investigación. 
CONTABILIDAD ELECTRÓNICA, ENFOQUE ESENCIAL PARA LA EDUCACIÓN CONTABLE

Muchos de los alumnos que estudian y trabajan a la vez, fortalece su formación académica y profesional, ya que los conocimientos que van adquiriendo en la universidad son complementados con los conocimientos que adquieren en su trabajo. Pero ¿Qué pasa con los estudiantes que no laboran aún?, es ahí donde yace la necesidad de enseñar el manejo de los software contables en la universidad.

Por último, respecto a qué tipos de software han utilizado en la universidad, el 39\% dijo usar el CONTPAQi, el 37\% señaló usar Aspel, el 19\% dijo usar solo el Microsoft Excel y el 5\% dijo haber ocupado algún otro (ver figura 5).

\section{De los siguientes software, ¿Cuál has utilizado en la universidad?}

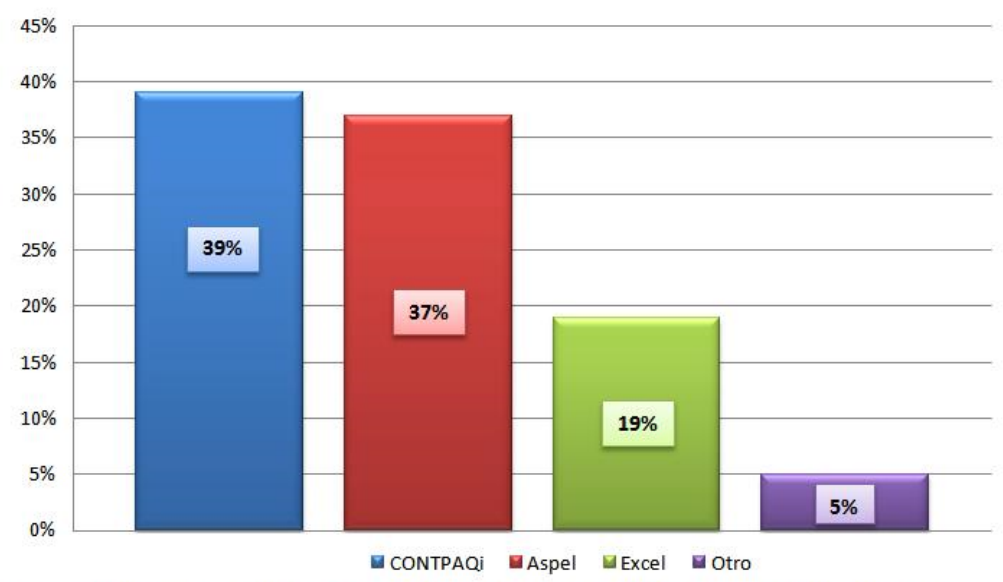

Figura 5. El software más utilizado en la universidad es el CONTPAQi, el cual es uno de los programas informáticos contables más populares entre las empresas.

Fuente: Resultado de la investigación.

La mayoría de los sistemas informáticos especializados en contabilidad, tienen similitudes en su funcionamiento, crean pólizas de diario, ingreso, egreso, preparan información para ser enviada al portal del SAT, entre otras funciones, es de gran importancia que el 56\% de los alumnos hayan usado el CONTPAQi y Aspel ya que a la hora de laborar en su campo, tendrán conocimiento previo de cómo manejar un software contable. 
CONTABILIDAD ELECTRÓNICA, ENFOQUE ESENCIAL PARA LA EDUCACIÓN CONTABLE

\section{DISCUSIÓN}

Actualmente, de acuerdo a los datos obtenidos de la investigación, el 57\% de los alumnos encuestados no tienen conocimiento de lo que es y lo que conlleva la contabilidad electrónica, el $29 \%$ sabe muy poco de este tema y el otro $14 \%$ afirma tener conocimiento.

La enseñanza de esta modalidad, muestra la inminente relación entre las novedades tecnológicas de la sociedad para ser impartida como disciplina académica, De la Torre (1994) señala que, el profesor es agente de la innovación y formará a través de la innovación.

Esto respalda la gran importancia que tienen los estudiantes en poseer conocimientos necesarios de la contabilidad electrónica para poder desenvolverse en un futuro en el ámbito laboral, haciendo actividades prácticas para su mayor aprendizaje.

A pesar de que el $75 \%$ de los estudiantes han usado algún software contable, el $57 \%$ de los alumnos no saben en qué consiste la contabilidad electrónica, esto puede ser debido a que, la ley es del año 2014 y los programas de estudio de la universidad no tenían considerado que se iba a convertir en una prioridad actualmente.

Bien se sabe que la teoría-conceptual (Teoría fundamentada) es el cimiento puro del discernimiento, pero actualmente por la demanda que hay en las empresas, los alumnos deben saber el conocimiento práctico. Acerca de esto, Donoso (1996) dice: "La lectura y los ejercicios siguen constituyendo el curriculum básico, aunque la tendencia es que los estudiantes tomen un papel mucho más activo".

Respecto a la enseñanza Lee (2017), nos enfatiza que, los docentes de contaduría de las universidades deberán contar con las capacidades siguientes: conocer métodos didácticos específicos de la Contabilidad Electrónica, la competencia tecnológica práctica y discernir el acompañamiento adecuado a los alumnos en el proceso formativo de la Contabilidad, en medios electrónicos.

El 68\% de los encuestados no saben manejar el portal web del SAT para algún tipo de trámite o declaración también tan solo el $45 \%$ de los alumnos apenas maneja un software contable, esto es debido a que la enseñanza se obstruye con la teoría dejando en una brecha prolongada la práctica. 
La contabilidad como cualquier otro proceso, está en constante cambio con el ambiente en el que lo envuelve, los educadores lo deben tener en cuenta al momento de impartir cátedra, para instruir a la nueva generación de profesionistas que se avecinan en pos de ellos, Martínez (2007) acerca de esto nos dice que el papel del profesor, como también el de quienes diseñan los programas curriculares de los diversos programas profesionales, deberá incluir un contexto, en el cual, el uso de las tecnologías de la información en las universidades, mantenga una relación en la que la comunicación se oriente a adquirir y a procesar conocimientos con sentido y pertinencia.

\section{CONCLUSIONES}

El contabilizar se ha mantenido en constante evolución desde sus inicios, así como el desarrollo de nuevas técnicas contables, pasando el tiempo para llegar a su mejoramiento. La contabilidad electrónica es el nuevo lenguaje del contador público, todo lo que antes se hacía en formatos de papel, hoy cambió gracias a la demanda de la tecnología.

Las obligaciones tributarias cumplimentadas por vía electrónica, por parte de los profesionales de la Contaduría, es un tema de gran relevancia hoy en día, en las universidades (Lee, 2017, p. 15).

La base de datos obtenida de la investigación realizada nos arroja que, actualmente los alumnos de la universidad están escasos de conocimiento acerca de la contabilidad electrónica, no saben su concepto, su incorporación fiscal y sus funciones, esto es algo que da qué pensar, ya que en la actualidad las empresas buscan personas preparadas conforme a las tecnologías.

Al ser el mecanismo que revolucionó la forma de contabilizar y con ello la recaudación de impuestos, es inherente y sustancial para los estudiantes de la carrera tener conocimiento de ello, como son: los softwares contables, su alcance y su importancia respecto a lo fiscal. Seltzer (2004) señala que, se debe enseñar todo aquello que sea útil al alumno como para que pueda desempeñarse tanto en el ámbito laboral en relación de dependencia, como para poder llevar adelante un emprendimiento propio.

Tanto el docente como el aprendizaje que se hallan en una interrelación educativa, no pueden quedar inmóviles y deben irse moldeando al ambiente en que opera, deben estar siempre latentes a la necesidades de la sociedad, al transcurrir el tiempo los sistemas se van 
CONTABILIDAD ELECTRÓNICA, ENFOQUE ESENCIAL PARA LA EDUCACIÓN CONTABLE

transformando y dejando nuevas parquedades, y la contabilidad no está exenta de este proceso.

Lee (2017), nos dice que la Licenciatura en Contaduría demanda que el egresado posea conocimientos de la contabilidad electrónica, para el desempeño profesional eficaz, eficiente y que impacte en un ambiente donde se transforma la sociedad industrial y se reclama un cambio para responder a las necesidades de la era digital.

El estudiante de la contabilidad como adquiriente del conocimiento, hace cargo al docente de otorgarle la correcta educación contable, el cual debe ir tomada de la mano de los nuevos enfoques tecnológicos y legales que puedan ayudarles en su etapa productiva laboral.

Con base a la información obtenida, se concluye con la necesidad que hay tanto en los docentes como en los comisionados de la elaboración del esquema de asignaturas de la Universidad Juárez Autónoma de Tabasco, División Académica de Ciencias Económico Administrativas de la Licenciatura en Contaduría, en actualizar el Plan de Estudios, ya que los alumnos de esta carrera se están viendo afectados con la falta de conocimiento y práctica, en materia de la contabilidad electrónica, con el objetivo de que los estudiantes, ostenten las capacidades contables, fiscales y tecnológicas para el registro de operaciones y presentación constante de la información financiera de las diversas entidades.

\section{REFERENCIAS BIBLIOGRÁFICAS}

Castro, P. (2015). 200 Preguntas y respuestas sobre contabilidad digital. México: Ediciones Fiscales Isef S.A. de C.V.

Ceballos, C. (2015). Contabilidad electrónica y su trasfondo fiscal. México: Ave Publicidad, S. A. de C. V.

CFF. (2013). Código Fiscal de la Federación. México: Cámara de Diputados H. Congreso de la Unión. Recuperado de: http://www.diputados.gob.mx/LeyesBiblio/index.htm

CINIF. (2016). Normas de Información Financiera. México: IMCP.

Corvera, P. (2014). El fisco se moderniza. Revista Veritas, Colegio de Contadores Públicos de México A.C. (1710), pp. 18-19. 
CONTABILIDAD ELECTRÓNICA, ENFOQUE ESENCIAL PARA LA EDUCACIÓN CONTABLE

Chávez, J. (2015). Contabilidad electrónica, un paso fuerte a la modernización contable. Ciencias Empresariales. (25), pp. 70-86.

De la Torre, S. (1994). Innovación curricular. España: Editorial Dykinson.

Donoso, J. (1996). Dirección "La docencia de la contabilidad a debate". España: Editorial Kronos.

Galindo, M. y Hernández, J. (2017). Estudio práctico de la contabilidad electrónica 2017. México: Editorial Isef Empresa Líder.

Gómez, M. (2007). Las insuficiencias disciplinares de los estándares internacionales de educación (IES) para contadores profesionales. Revista Lúmina. 24(08), pp. 24-42.

Granada, H. (1984). La teoría: su estructura e importancia en la investigación científica. Revista de psicología, 2(1-2).

Lang, V. (2015). Globalización y contabilidad. Revista Veritas, Colegio de Contadores Públicos de México A.C. (1727), pp. 8-10.

Lara, E. (2006). Principios básicos de contabilidad. México: Trillas S.A de C.V.

Lee, P. (2017). La importancia de la contabilidad electrónica en el currículum. Realidad y Reflexión $17 . \quad$ (45), pp. 8-17. $\quad$ Recuperado de https://www.lamjol.info/index.php/RyR/article/view/4415/4172

LISR. (2013). Ley del Impuesto sobre la Renta. México: Cámara de Diputados H. Congreso de la Unión. Recuperado de: http://www.diputados.gob.mx/LeyesBiblio/index.htm

Marín, Á. (2014). Ordenamientos mercantiles y civiles, implicaciones legales de la contabilidad. Revista Veritas, Colegio de Contadores Públicos de México A.C. (1716), pp. 44-45.

Martínez, G. (2007). La educación contable en el nuevo contexto de significación. Contaduría Universidad de Antioquia. (50), pp. 43-76.

Pérez, J. y Fol, R. (2016). Contabilidad electrónica y su envió a través del portal del SAT. México: Tax Editores Unidos. 
CONTABILIDAD ELECTRÓNICA, ENFOQUE ESENCIAL PARA LA EDUCACIÓN CONTABLE

Secretaría de Hacienda y Crédito Público. (2015). La nueva administración tributaria en México 2a Edición. México: Amexipac. Recuperado de: http://www.sat.gob.mx/sala_prensa/comunicados_nacionales/2015/agosto/Documents/L aNuevaAdministracionTributariaenMexico.pdf

Seltzer, J. (2004). Contabilidad y docencia. Revista iberoamericana de educación. (33), pp. 112. 